\title{
Influence of fluctuations in actin structure on myosin V step size
}

\author{
Andrej Vilfan \\ J. Stefan Institute, Jamova 39, 1000 Ljubljana, Slovenia
}

(Dated: 1.5 .2005$)$

\begin{abstract}
We study the influence of disorder in the helical actin structure on the myosin V step size, predicted from the elastic lever arm model. We show that fluctuations of \pm 5 degrees per actin subunit, as proposed by Egelman et al., significantly alter the distribution of step sizes and improve the agreement with experimental data.
\end{abstract}

\section{Introduction}

Myosin V is a motor protein from the myosin superfamily, involved in various intracellular transport processes [1]. It is a processive motor [2], which means that a single protein molecule can transport cargoes along actin filaments over distances of several micrometers. It is dimeric, consisting of two identical heads, each attached to a lever arm, joined with each other through a tail domain. It achieves processivity by alternately binding its two heads to an actin filament and thus walking in a hand-over-hand fashion [3]. Its step size is roughly determined by the periodicity of the actin filament, which is about $36 \mathrm{~nm}$.

In comparison with muscle myosin (myosin II), two major adaptations are found in myosin V: a longer leverarm, measuring around $26 \mathrm{~nm} \mathrm{[4]} \mathrm{and} \mathrm{a} \mathrm{slower} \mathrm{release} \mathrm{of}$ ADP. The duty cycle of each head is otherwise similar [5]: the head binds to actin in the ADP.Pi state, first releases $\mathrm{Pi}$ and performs a large conformational change (power stroke), then releases ADP and performs a smaller conformational change, and finally binds a new ATP molecule and detaches from actin.

In a recent article [6], we have developed a model for myosin $\mathrm{V}$, based on the elasticity of the lever arm. It assumes that the lever arm is stiffly anchored in the head, but the direction depends on the chemical state of that head. The distal ends of the lever arms are connected together through a flexible hinge, which represents the only means of "communication" between the heads. By calculating the bending energies in all relevant dimer states and its influence on transition rates, we have shown that the elastic lever-arm model explains the coordinated hand-over-hand motility by showing that the lead head is not likely to bind to actin before the trail head undergoes the major power stroke and that it cannot commit its power stroke before the trail head unbinds. The model also quantitatively reproduces the measured force-velocity relations and shows how the run length (the average distance a motor runs processively before it dissociates from actin) could be used to determine some kinetic rates. Although the original model reproduces the step size corresponding to about one halfturn of the actin helix (13 actin subunits), there is a small but significant deviation between the predicted distribution of step sizes (mainly on the 13th subunit, with a side peak on the 11th) and the statistics obtained from elec-

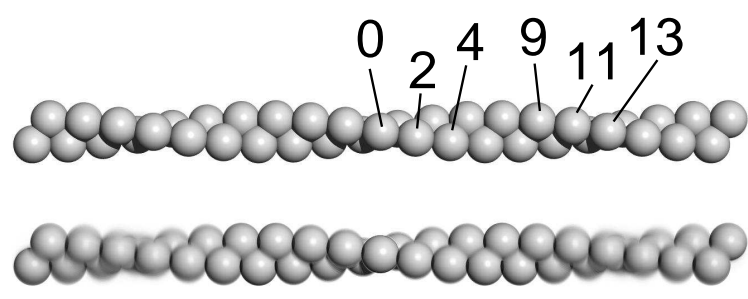

FIG. 1: The regular 13/6 helical model of an actin filament (top), and the $28 / 13$ helix with $\pm 5^{\circ}$ fluctuations per subunit (bottom). The subunits are shown blurred, with a relative intensity corresponding to their statistical weight. The orientation of the central subunit (denoted as "0") is always kept fixed.

tron microscopy (EM) studies [7], which show the main peak on the 13th subunit and two significant side peaks on the 11 th and the 15 th subunit, whereby the 15 th is stronger than the 11th.

The purpose of the current paper is to extend the present model by including a more precise description of the actin structure, which could potentially explain the current deviation. The central modification will be to take into account torsional fluctuations in the actin structure. So far we have used the assumption that the actin monomers are arranged on a helix with a periodicity of 13 actin subunits (also called a 13/6 helix, because the rotation of each next subunit is $\frac{6}{13} \times 360^{\circ}$. However, it has been known for some time that the actin filaments have a variable, fluctuating twist [8]. The nature and the dynamics of these fluctuations are not yet entirely understood, but it seems that the azimuthal orientation of each subunit can fluctuate about $\pm 5^{\circ}$ with respect to its neighbors $[9]$. There is some evidence that the dynamics of these fluctuations is rather slow 10, 11], with a characteristic time of the order of seconds. This would imply that the twist has discrete states with a considerable energy barrier between them. The average twist per subunit was found to be about $167^{\circ}$, slightly more than in the $13 / 6$ helix, where it would be $166.15^{\circ}$ (Fig. (1).

Based on many experimental and theoretical studies [3, 6, 12, 13], the following picture of the working cycle of dimeric myosin $\mathrm{V}$ has now found broad consensus (Fig. 2). The major part of the dimeric cycle is spent in the state with both heads binding ADP. The trail head 
TABLE I: Model parameters

\begin{tabular}{lll}
\hline Lever arm length & $L$ & $26 \mathrm{~nm}$ for 6IQ (WT) \\
Lever arm start (radial) & $R$ & $8 \mathrm{~nm}$ \\
Lever arm start (longitudinal) & $\delta_{\text {ADP.Pi }}$ & $0 \mathrm{~nm}$ \\
& $\delta_{\text {ADP }}$ & $3.5 \mathrm{~nm}$ \\
Angle ADP.Pi & $\phi_{\text {ADP.Pi }}$ & $115^{\circ}$ \\
Angle ADP & $\phi_{\text {ADP }}$ & $50^{\circ}$ \\
Bending modulus & $E I$ & $1500 \mathrm{pN} \mathrm{nm}^{2}$ \\
Average helix twist per subunit & $\theta^{0}$ & $167.14^{\circ}$ \\
Twist fluctuations & $\theta^{\prime}$ & $5^{\circ}$ \\
Helix rise per subunit & $a$ & $2.75 \mathrm{~nm}$ \\
\hline
\end{tabular}

is in the post-powerstroke and the lead head likely in the pre-powerstroke state [4, 6, 14], although the latter has still been controversial recently (cf. ref. 15]). Then the trail head releases ADP, binds a new ATP molecule and unbinds from actin. With the trail head released, the lead head is now free to undergo a power-stroke, whereupon the former trail head binds in the lead position (one step ahead in the direction of motion) and releases $\mathrm{Pi}$. This brings the dimer to the original state, however one step further toward the actin plus end and with one ATP molecule hydrolyzed.

\section{Results}

To calculate the step size, which is the main objective of this article, the relevant transition is the binding of the lead head (in the ADP.Pi state) while the trail head is in the forward-leaning ADP conformation. Let us denote the binding rate to the site $i$ (counted from the trail head, which is bound to site 0 ) with $k_{+A(i)}$ and the rate of the reverse (unbinding) process as $k_{-A(i)}$. From the detailed balance we know that the ratio of these rates has to obey

$$
\frac{k_{+A(i)}}{k_{-A(i)}}=\frac{k_{+A}^{0}}{k_{-A}^{0}} \exp \left[-\frac{\Delta U+F \Delta x}{k_{B} T}\right]
$$

where $\Delta U$ denotes the increase in elastic energy upon binding and $F \Delta x$ denotes the work done against the applied load. $k_{+A}^{0}$ and $k_{-A}^{0}$ represent the binding and unbinding rates of a head that binds without any elastic distortions in the lever arm. Because the persistence length of the lever arms is significantly longer than their actual length, we neglect thermal fluctuations and the corresponding entropic contributions to the total free energy. In the following we will also concentrate on the case of an unloaded motor $(F=0)$. In this case the bending energy in the initial state is 0 and $\Delta U$ is equal to the elastic energy in the double-bound state. All model parameters are summarized in Table $\square$ and Fig. 3 The geometric parameters were obtained from published EM studies [4, 7]. The bending modulus of the lever arm $(E I)$ was estimated [ $[$ ] based on a lower boundary provided by the fact that a myosin $\mathrm{V}$ molecule is able to perform regular steps against loads of at least $1.8 \mathrm{pN}[16]$.

The elastic energy of a certain dimer conformation is calculated in the following way (which is described in more detail in Ref. [a] $)$. A lever arm starting point is positioned at

$$
\mathbf{x}^{0}=\left(\begin{array}{c}
i a+\delta \\
R \sin \left(\theta_{i}\right) \\
R \cos \left(\theta_{i}\right)
\end{array}\right)
$$

and its initial tangent is

$$
\hat{t}^{0}=\left(\begin{array}{c}
\cos (\phi) \\
\sin (\phi) \sin \left(\theta_{i}\right) \\
\sin (\phi) \cos \left(\theta_{i}\right)
\end{array}\right) .
$$

Other conditions state that the length of the lever arm from the starting point to the joint has to be $L$ and that the endpoints of both lever arms coincide. The bending energy is then given as

$$
U=\sum_{j=1}^{2} \int_{0}^{L} \frac{E I}{2}\left(\frac{d \hat{t}_{j}}{d s}\right)^{2} d s
$$

where $E I$ denotes the bending modulus and $\frac{d \hat{t}}{d s}$ the local curvature of a lever arm. The summation index $j$ runs over both lever arms. The shape of the dimeric molecule is determined numerically in a way that minimizes $U$.

There are two possibilities concerning the reversibility of the binding process. One possibility is that the lead head binds to a site and does not detach before the next power stroke. In this case, the probability that it binds to the site $i$ is

$$
P_{i}=\frac{k_{+A(i)}}{\sum_{j} k_{+A(j)}}
$$

If, however, the lead head in the ADP.Pi (weakly bound) state is allowed to detach and re-attach several times, the probability to find it on the site $i$ is given by the equilibrium distribution,

$$
P_{i}=\frac{k_{+A(i)} / k_{-A(i)}}{\sum_{j} k_{+A(j)} / k_{-A(j)}} .
$$

So far, no experimental data are available about the strain dependence of the detachment rate, $k_{-A}$. However, as with most binding processes one can expect that the activation point for the binding transition is closer to the bound state, and therefore the strain-dependence of $k_{-A}$ is weaker than that of $k_{+A}$. We therefore neglect the strain-dependence of detachment rates and assume that the Boltzmann factor in Eq. (11) only influences $k_{+A(i)}$. If the binding process is reversible, $P_{i}$ (Eq. 6) becomes independent of this assumption in any case.

To describe the fluctuations in the actin helix, we will use the coefficients $a_{i}$, which can assume the values -1 , 


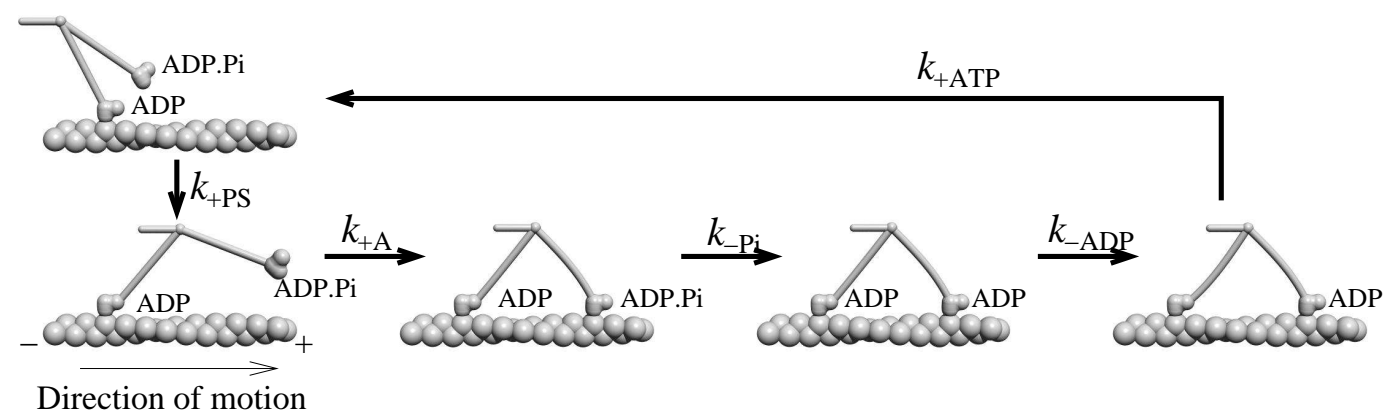

FIG. 2: The most likely duty cycle of the dimeric myosin V. A head in the ADP state undergoes the power-stroke, then the second head containing ADP.Pi binds in the lead position, it releases Pi, then the rear head releases ADP (time limiting), binds a new ATP molecule and detaches from actin and the cycle repeats with exchanged roles of the two heads, however with the dimer having proceeded about one actin period further.
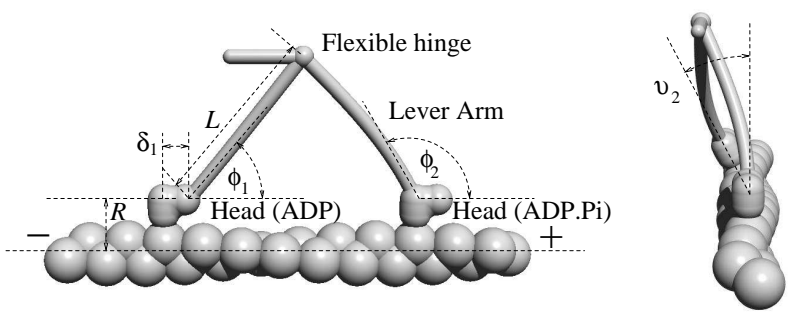

FIG. 3: The geometry of a dimeric myosin V molecule, after the lead head has bound to a site 13 subunits in front of the trail head.

0 or 1 . This corresponds to the assumption that each subunit can have three orientations relative to its left neighbor [10]. The twist between the subunits $i-1$ and $i$ is then $\theta_{i}-\theta_{i-1}=\theta^{0}+a_{i} \theta^{\prime}$, and the total twist of subunit $i$ relative to subunit 0 is $\theta_{i}=i \theta^{0}+\left(a_{1}+a_{2}+\ldots a_{i}\right) \theta^{\prime}$.

Another important question is the dynamics of the actin fluctuations. Let us denote the probability that the helix is in the state with angles $\theta^{0}+a_{1} \theta^{\prime}, \theta^{0}+a_{2} \theta^{\prime}$, ... with

$$
P_{a_{1}, a_{2}, \ldots, a_{i}}=\left(\frac{1}{3}\right)^{i}
$$

If the fluctuations are fast in comparison with the attachment rate, the probability that the head binds to site $i$ is proportional to the attachment rate in each configuration, weighted by the probability of that configuration and summed over all possible configurations:

$P_{i}^{f}=\frac{\sum_{a_{1}, a_{2}, \ldots a_{i}} P_{a_{1}, a_{2}, \ldots, a_{i}} e^{-U_{i}\left(\theta^{0} i+\theta^{\prime}\left(a_{1}+a_{2}+\ldots a_{i}\right)\right) / k_{B} T}}{\sum_{j} \sum_{a_{1}, a_{2}, \ldots a_{j}} P_{a_{1}, a_{2}, \ldots, a_{j}} e^{-U_{j}\left(\theta^{0} j+\theta^{\prime}\left(a_{1}+a_{2}+\ldots a_{j}\right)\right) / k_{B} T}}$

Here $U_{i}(\theta)$ denotes the elastic energy in a state where the lead head is bound $i$ subunits in front of the trail head and the helical twist between these two subunits is $\theta$. A simplified expression for $P_{i}^{f}$ is derived in the Appendix.
If, on the other hand, the fluctuations are slow, the probability is given by the ensemble-average of all helix conformations:

$$
P_{i}^{s}=\sum_{a_{1}, a_{2}, \ldots} P_{a_{1}, a_{2}, \ldots, a_{n}} \frac{e^{-U_{i}\left(\theta^{0} i+\theta^{\prime}\left(a_{1}+a_{2}+\ldots a_{i}\right)\right) / k_{B} T}}{\sum_{j} e^{-U_{j}\left(\theta^{0} j+\theta^{\prime}\left(a_{1}+a_{2}+\ldots a_{j}\right)\right) / k_{B} T}}
$$

Here $n$ denotes the maximum index of a site that still has a non-negligible binding probability. In the following, we will calculate the results for both scenarios, even though we consider the slowly fluctuating scenario more realistic.

A numerical evaluation of the probability distributions $P_{i}^{s}$ and $P_{i}^{f}$ for four different lever arm lengths, corresponding to mutants with $2,4,6$ (wild type) and 8 IQ motifs in the lever arm shows a notable difference in comparison with results on the $13 / 6$ helix (Fig. 4).

To check the effects of uncertainties in other model parameters on our results, we have tried three modifications: we have introduced an additional azimuthal component of the power-stroke (meaning that after Pi release, the lever arm makes an additional swing of $20^{\circ}$ to the right, while its starting point remains the same), we have changed the lever arm angle $\phi$ in the pre-powerstroke state and we have changed the lever arm stiffness. The results are summarized in TableII None of the tested modifications makes an improvement with regard to agreement with experimental data as compared to the basic model.

Another assumption we made in our model is that the lever arm anchoring is stiff in every state. While this assumption is justified for long lever arms, the effect of compliance in the converter domain could play an important role for short lever arms. Results on skeletal muscle myosin (with short lever arms) indeed show that at least half of the compliance is in the converter domain [17]. Therefore some care has to be taken when interpreting the results for 2IQ lever arms. 
TABLE II: Average step size for the basic model and the following variations: (i) additional azimuthal power stroke, (ii) different lever arm angle in the pre-powerstroke conformation $\left(\phi_{A D P . P i}=135^{\circ}\right.$ instead of $\left.115^{\circ}\right)$ and (iii) a softer lever-arm $\left(E I=500 \mathrm{pN} \mathrm{nm}^{2}\right.$ instead of $\left.1500 \mathrm{pN} \mathrm{nm}^{2}\right)$. All data are based on the slow-fluctuation scenario.

\begin{tabular}{lllll} 
Lever arm length & basic model & $\Delta \theta=20^{\circ}$ & $\phi_{A D P . P i}=135^{\circ}$ & $E I=500 \mathrm{pN} \mathrm{nm}^{2}$ \\
\hline $2 \mathrm{IQ}$ & 2.0 & 2.28 & 2.6 & 2.1 \\
$4 \mathrm{IQ}$ & 8.1 & 4.5 & 11.7 & 7.9 \\
$6 \mathrm{IQ}$ & 13.4 & 14.1 & 14.0 & 13.9 \\
$8 \mathrm{IQ}$ & 14.1 & 15.1 & 14.7 & 13.2 \\
\hline
\end{tabular}

(A)

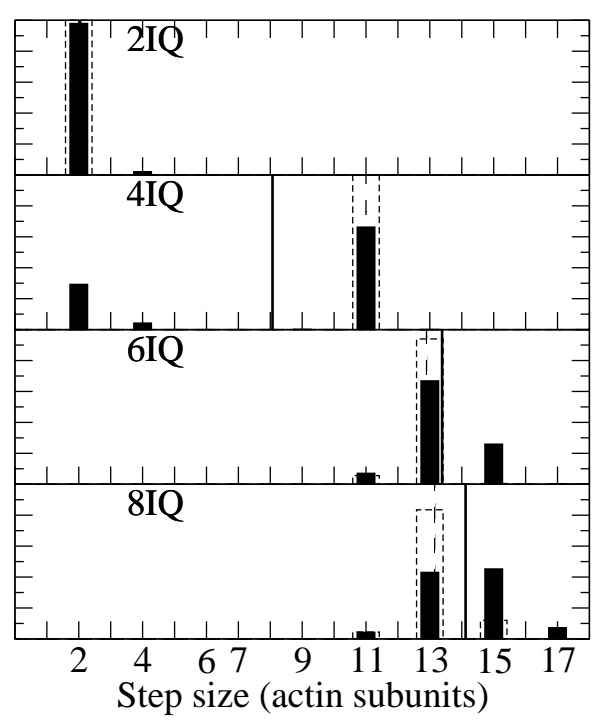

(B)

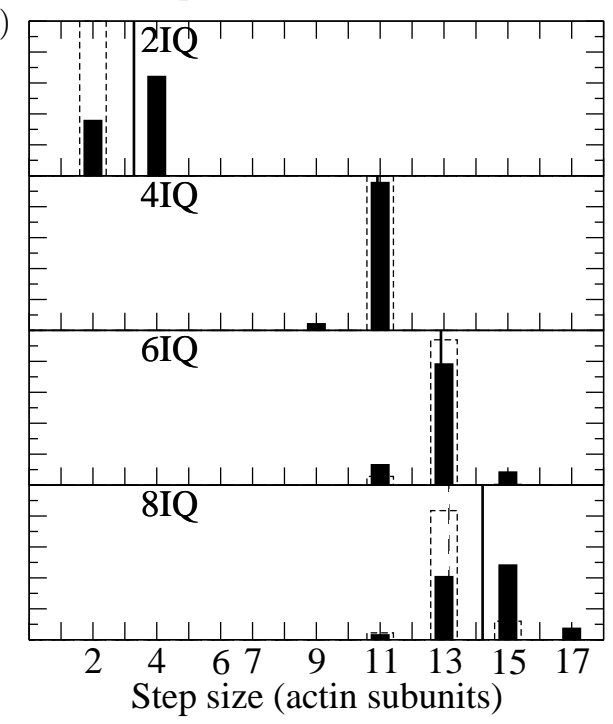

FIG. 4: The probabilities that the lead head binds to site $i$ in the model with slow fluctuations $\left(P_{i}^{s}, \mathrm{~A}\right)$ and fast fluctuations $\left(P_{i}^{f}, \mathrm{~B}\right)$. The four diagrams are for different lever arm lengths (2IQ: 10nm; 4IQ: 18nm; 6IQ: 26nm; 8IQ: 34nm). The vertical lines in each diagram show the average position $\langle i\rangle$. The dashed lines represent results on a rigid $13 / 6$ helix, as used in previous calculations.

\section{Discussion}

The results for the slowly fluctuating scenario show a significantly improved agreement with available experiments [7] than the previous model, based on a stiff $13 / 6$ helix. In particular, the observation that the probability for 15 subunit steps is about twice as high as for 11 subunit steps is well reproduced. The average step size of 13.4 actin subunits (on a helix with a half-pitch of 14) also means that the molecule makes a left-handed rotation of about $8^{\circ}$ per step, or $0.2^{\circ}$ per $\mathrm{nm}$ traveled. This means that a freely walking myosin $\mathrm{V}$ makes one revolution around the actin filament every $1.7 \mu \mathrm{m}$, well consistent with a value of $2.2 \mu \mathrm{m}$, measured by Ali and co-workers 18]. Note that their interpretation (based on the assumption of a $13 / 6$ helix) is that the steps represent a mixture of 11 and 13 subunit lengths. However, on a $28 / 13$ helix, data become consistent with a mixture including 15 subunit steps, which are even more frequent than 11 subunit steps.

The third experiment our model should be tested against is the dependence of the step size on the lever arm length. Purcell and co-workers [19] used optical tweezers to determine the step size of different dimeric constructs. For wild-type myosin V (6 IQ domains), they measured a step size of $35 \mathrm{~nm}$. For $4 \mathrm{IQ}$ mutants they measured $24 \mathrm{~nm}$ or 8.7 actin subunits, and for 1IQ mutants $5 \mathrm{~nm}$ or 2 subunits. Also in this respect the current model (predicting 13.4, 8 and 2 subunit steps, respectively) noticeably improves the agreement with experimental data. The previous model, based on a stiff helix, predicted a more abrupt step size change between 2 and 4IQ mutants.

\section{Appendix}

In Eq. (87) the summation over coefficients $a_{1}, \ldots, a_{i}$ can be carried out while keeping their sum constant $\left(s_{i}\right)$. This way we obtain the probability that the subunit $i$ is oriented at angle $\theta^{0} i+\theta^{\prime} s_{i}$ :

$$
\begin{aligned}
P_{i, s_{i}} & =\sum_{a_{1}, a_{2}, \ldots, a_{i} ; a_{1}+a_{2}+\ldots+a_{i}=s_{i}} P_{a_{1}, a_{2}, \ldots, a_{i}} \\
& =\frac{1}{3^{i}} \sum_{k ;\left|s_{i}\right| \leq k \leq\left(i+\left|s_{i}\right|\right) / 2}\left(\begin{array}{c}
i \\
k
\end{array}\right)\left(\begin{array}{c}
i-k \\
k-s
\end{array}\right)
\end{aligned}
$$


Then expression (8) can be simplified to

$$
P_{i}^{f}=\frac{\sum_{s_{i}=-i}^{i} P_{i, s_{i}} e^{-U_{i}\left(\theta^{0} i+\theta^{\prime} s_{i}\right) / k_{B} T}}{\sum_{j} \sum_{s_{j}=-j}^{j} P_{j, s_{j}} e^{-U_{j}\left(\theta^{0} j+\theta^{\prime} s_{j}\right) / k_{B} T}} .
$$

For large values of $i$ the central limit theorem can be applied and the distribution $P_{i, s_{i}}$ becomes Gaussian.

\section{Acknowledgment}

This work was supported by the Slovenian Office of Science (Grants No. Z1-4509-0106-02 and P0-0524-0106).
[1] Reck-Peterson, S. L.; Provance, Jr., D. W.; Mooseker, M. S.; Mercer, J. A. Biochim. Biophys. Acta 2000, 1496, 36-51.

[2] Mehta, A. D.; Rock, R. S.; Rief, M.; Spudich, J. A.; Mooseker, M. S.; Cheney, R. E. Nature 1999, 400, 590593.

[3] Vale, R. D. J. Cell. Biol. 2003, 163, 445-450.

[4] Burgess, S.; Walker, M.; Wang, F.; Sellers, J. R.; White, H. D.; Knight, P. J.; Trinick, J. J. Cell. Biol. 2002, 159, 983-991.

[5] De La Cruz, E. M.; Ostap, E. M. Curr. Opin. Cell Biol. 2004, 16, 61-67.

[6] Vilfan, A. Biophys. J. 2005, 88, 3792-3805.

[7] Walker, M. L.; Burgess, S. A.; Sellers, J. R.; Wang, F.; Hammer, J. A.; Trinick, J.; Knight, P. J. Nature 2000, 405, 804-807.

[8] Egelman, E. H.; Francis, N.; DeRosier, D. J. Nature 1982, 298, 131-135.

[9] Egelman, E. H.; DeRosier, D. J. Biophys. J. 1992, 63, 1299-1305.

[10] Egelman, E. H. Structure 1997, 5, 1135-1137.
[11] Orlova, A.; Egelman, E. H. Biophys. J. 2000, 78, 21802185.

[12] Rief, M.; Rock, R. S.; Mehta, A. D.; Mooseker, M. S.; Cheney, R. E.; Spudich, J. A. Proc. Natl. Acad. Sci. USA 2000, 97, 9482-9486.

[13] Rosenfeld, S. S.; Sweeney, H. L. J. Biol. Chem. 2004, 279, 40100-40111.

[14] Snyder, G.; Syed, S.; Goldman, Y.; Selvin, P. Biophys. J. 2005, 88, 2450-Pos, Part 2 Suppl. S.

[15] Snyder, G. E.; Sakamoto, T.; Hammer, J. A.; Sellers, J. R.; Selvin, P. R. Biophys. J. 2004, 87, 1776-1783.

[16] Mehta, A. D.; Rock, R. S.; Rief, M.; Spudich, J. A.; Mooseker, M. S.; Cheney, R. E. Nature 1999, 400, 590.

[17] Köhler, J.; Winkler, G.; Schulte, I.; Scholz, T.; McKenna, W.; Brenner, B.; Kraft, T. Proc. Natl. Acad. Sci. USA 2002, 99, 3557-3562.

[18] Ali, M. Y.; Uemura, S.; Adachi, K.; Itoh, H.; Kinosita Jr, K.; Ishiwata, S. Nat. Struct. Biol. 2002, 9, 464-467.

[19] Purcell, T. J.; Morris, C.; Spudich, J. A.; Sweeney, H. L. Proc. Natl. Acad. Sci. USA 2002, 99, 14159-14164. 